\title{
Different neural pathways linking personality traits and eudaimonic well-being: a resting-state functional magnetic resonance imaging study
}

\author{
Feng Kong • Ling Liu • Xu Wang • Siyuan Hu • Yiying Song • Jia Liu
}

Published online: 21 November 2014

(C) Psychonomic Society, Inc. 2014

\begin{abstract}
Eudaimonic well-being (EWB) is the fulfillment of human potential and a meaningful life. Previous studies have shown that personality traits, especially extraversion, neuroticism, and conscientiousness, significantly contribute to EWB. However, the neurobiological pathways linking personality and EWB are not understood. Here, we used restingstate functional magnetic resonance imaging (rs-fMRI) to investigate this issue. Specifically, we correlated individuals' EWB scores with the regional fractional amplitude of lowfrequency fluctuations (fALFF) of the brain, and then examined how personality traits predicted EWB-related spontaneous brain activity. We found that EWB was positively correlated with the AALFF in the right posterior superior temporal gyrus (pSTG) and thalamus, and negatively correlated with the strength of the thalamic-insular connectivity. More importantly, we found that personality traits influenced EWB in different ways. At the regional level, the fALFF in the pSTG and thalamus mediated the effects of neuroticism and extraversion on EWB, whereas the thalamus mediated the effect of conscientiousness on EWB. At the functional connectivity level, the thalamic-insular connectivity only mediated the effect of neuroticism on EWB. Taken together, our study provides the first evidence that EWB is associated with personality traits through different neural substrates.
\end{abstract}

F. Kong $\cdot$ L. Liu $\cdot$ X. Wang $\cdot$ Y. Song

State Key Laboratory of Cognitive Neuroscience and Learning \& IDG/McGovern Institute for Brain Research, Beijing, China

F. Kong $\cdot$ L. Liu $\cdot$ X. Wang $\cdot$ Y. Song

Center for Collaboration and Innovation in Brain and Learning

Sciences, Beijing, China

S. Hu J J. Liu $(\square)$

School of Psychology, Beijing Normal University, Beijing, China

e-mail: liujia@bnu.edu.cn
Keywords Eudaimonic well-being · Personality · Extraversion $\cdot$ Conscientiousness $\cdot$ Neuroticism $\cdot$ fALFF

\section{Introduction}

In the past few decades, the concept of eudaimonic well-being (EWB) has drawn increasing attention in the scientific literature. EWB was first proposed in Aristotle's Nicomachean Ethics, which has been conceptualized as the realization of valued human potential (Ryan \& Deci, 2001) and a purposeful and meaningful life (Ryff, 1989). The concept is often differentiated from hedonic well-being (hedonia), which reflects the transient experience of pleasure (Ryan \& Deci, 2001). Recently, researchers have begun to explore how individuals increase their own levels of EWB, which to a certain extent depends on their personality characteristics (Steel et al., 2008). Several studies have demonstrated that the importance of personality characteristics, especially traits from the fivefactor model (FFM; Costa \& McCrae, 1992) of personality in EWB (Abbott, Croudace, Ploubidis, Kuh, Richards, \& Huppert, 2008; Augusto Landa, Martos, \& Lopez-Zafra, 2010; Garcia, 2011; Grant, Langan-Fox, \& Anglim, 2009; Lyubomirsky, Sheldon, \& Schkade, 2005; Schmutte \& Ryff, 1997; Steel, Schmidt, \& Shultz, 2008). Although there appears to be an important relationship between personality and EWB, the neurobiological pathways linking personality and EWB are not yet understood.

The relationship between personality and well-being has long been investigated. As early as 1989, Headey and Wearing proposed a dynamic equilibrium model of well-being that emphasizes the importance of personality in well-being. According to this model, when recent positive or adverse life events result in deviation from normal patterns causing wellbeing levels to change, personality characteristics will restore well-being to its normal level of equilibrium. Several studies 
have shown that personality significantly contributes to EWB (Abbott et al., 2008; Augusto Landa et al., 2010; Garcia, 2011; Grant et al., 2009). For instance, personality traits such as neuroticism, extraversion, and conscientiousness account for $52 \%$ of the variance in adolescents' EWB (Garcia, 2011) and approximately $16-27 \%$ of the variance in adults' EWB (Augusto Landa et al., 2010). Furthermore, Abbott et al. (2008) have found that extraversion and neuroticism in adolescence (age 16 years) account for roughly $20 \%$ of the variance in EWB in early adulthood (age 26 years). Taken together, these findings suggest that personality traits, especially neuroticism, extraversion, and conscientiousness, are important contributors to EWB. In addition, previous studies have indicated that the "big five" personality traits (openness, conscientiousness, extraversion, agreeableness, and neuroticism) are relatively independent from each other (Costa \& McCrae, 1995); therefore, they may be associated with EWB through different neurobiological mechanisms.

Previous neuroimaging studies have begun to investigate the neural basis of EWB at the regional level and found that EWB is involved in multiple brain regions including the temporal, insular, prefrontal, and limbic cortices (Heller et al., 2013; Lewis, Kanai, Rees, \& Bates, 2013; Luo, Huang, Yang, Li, Liu, \& Wei, 2014; van Reekum et al., 2007). For example, structurally, higher EWB is associated with larger gray matter volume (GMV) in the insula (Lewis et al., 2013). Functionally, higher scores on EWB or related measures (e.g., happiness) are associated with increased activities of the superior temporal lobe and decreased activities of the thalamus at rest (Luo et al., 2014), and associated with increased or sustained activities of the prefrontal cortex in response to affective stimuli (Heller et al., 2013; van Reekum et al., 2007). In this study we tried to use restingstate functional magnetic resonance imaging (rs-fMRI) to explore the neural correlates of EWB at both the regional and the functional connectivity levels in a unified way, and examine how its neural correlates are differently associated with the five basic personality traits. Studies using the rs-fMRI method are particularly suitable for exploring the neural correlates of EWB because it may be difficult to include the cognitive processes involving the whole range of EWB in the short tasks characteristic of fMRI studies. Furthermore, the rs-fMRI method might better tap into tonic, long-lasting components of brain function.

In this study, we focused on the low frequency fluctuations (LFFs, 0.01-0.10 Hz) in the blood oxygen level-dependent (BOLD) signal at rest, which are related to spontaneous neuronal activities (Biswal, 2012; Fox and Raichle, 2007; Raichle, 2010). Specifically, we measured two different aspects of low frequency BOLD oscillations: the fractional amplitude of low frequency fluctuations (fALFF) reflecting regional properties of intrinsic brain dynamics and the restingstate functional connectivity (RSFC) that provides information about synchronization between spatially distinct brain regions (Fox and Raichle, 2007; Zou et al., 2008). The fALFF is more of an activation-like measure (i.e., in which there is no manipulation of task state or transient evokedresponses), whereas the RSFC indexes task-independent connectivity. Previous studies have shown that fALFF and RSFC reflect neuronal signals and are behaviorally relevant (Biswal, 2012; Fox and Raichle, 2007; Raichle, 2010). Both aspects have been found to identify populations with various types of neuropsychiatric disorders such as attention-deficit hyperactivity disorder (ADHD; Li et al., 2014), Alzheimer's disease (Liu et al., 2008), major depressive disorder (Jiao et al., 2011; Wang et al., 2012; Liu et al., 2013), mild cognitive impairment (Han et al., 2011; Zhao et al., 2014), and schizophrenia (Hoptman et al., 2010; Yu et al., 2014). In addition, several recent studies have shown healthy individuals' fALFF and RSFC are associated with individual differences in executive control (Mennes et al., 2011; Xu et al., 2014), face processing (Zhu, Zhang, Luo, Dilks, \& Liu, 2011), object color processing (Wang et al., 2013), emotional intelligence (Cox et al, 2012; Takeuchi et al., 2013), and personality traits (Wei et al., 2014). These findings indicate that fALFF and RSFC can effectively reflect the neural mechanisms for cognitive and emotional functions.

In the current study we extended the previous studies in three ways. First, we employed the most widely used EWB questionnaire (Ryff et al., 2007) to measure the EWB of healthy individuals $(n=285)$. Because EWB represents the subjective experience of an individual, self-report is arguably the most suitable assessment (Ryff, 1989). Second, we identified the neural correlates of EWB at both regional and functional connectivity levels. At the regional level, we performed a correlation analysis between EWB and the fALFF to identify regions associated with individual differences in EWB. At the functional connectivity level, we computed the RSFC between the core region(s) identified above and other voxels in the brain, and then correlated the RSFC strength with EWB. Finally, to examine how the five basic personality traits are differently associated with the neural correlates of EWB, we performed multiple mediation analyses to test whether and how intrinsic brain activity mediates the associations between personality traits and EWB.

\section{Methods}

\section{Participants}

Two hundred and eighty-six healthy university students (155 females; mean age $=21.55$ years, standard deviation $[\mathrm{SD}]=$ 1.01 ) with no history of neurological or psychiatric disorders were recruited from Beijing Normal University (BNU) as paid participants. Both behavioral and magnetic resonance imaging 
(MRI) protocols were approved by the Institutional Review Board of Beijing Normal University. Written informed consent was obtained from all the participants prior to the study.

\section{Behavioral tests}

EWB was assessed using the 42-item Scales of Psychological Well-being (SPWB; Ryff et al., 2007), which is a highly reliable measure for assessing EWB (Ryff, 1989, Ryff et al., 2007). The SPWB is composed of six dimensions (autonomy, environmental mastery, self-acceptance, positive relations with others, purpose in life, and personal growth), with sample items such as "Some people wander aimlessly through life, but I am not one of them" and "I think it is important to have new experiences that challenge how you think about yourself and the world." Each dimension was measured with seven items and participants responded to each item using a 6-point Likert scale with response options ranging from strongly disagree to strongly agree. Ryff and colleagues (e.g., Ryff \& Keyes, 1995) reported that a single EWB factor may also explain associations among these six constructs; therefore, a mean score across the six subscales was calculated as an overall score to index EWB, with a higher score indicating higher EWB. The scale has been found to have good validity and internal reliability $(\alpha=0.73-0.87)$ in young Chinese adults (Wan, Zheng, \&Yu, 2010). Consistent with previous studies in Western cultures (Gallagher, Lopez, \& Preacher, 2009; Lindfors, Berntsson, \& Lundberg, 2006; Ryff, 1989), the correlations between the EWB subscales observed in the present study were moderate (ranging from .20 to .66). In the present study, SPWB also exhibited adequate internal reliability $(\alpha=0.92)$.

The "big five" personality dimensions were assessed using the Revised NEO Personality Inventory (NEO-PI-R; Costa \& McCrae, 1992). The NEO-PI-R is a 120 -item self-report questionnaire based on the five-factor model of personality (Costa \& McCrae, 1990; Costa \& McCrae, 1995). Each personality dimension was measured with 24 items and the answering categories ranged from 1 (strongly disagree) to 5 (strongly agree). The NEO-PI-R provides summary scores for the five different dimensions of personality as mentioned above: neuroticism, extraversion, openness to experience, agreeableness, and conscientiousness. In the present study, Cronbach's $\alpha$ of the NEO-PI-R scales ranged from .71 to .87 , indicating that these five personality dimensions exhibited adequate internal reliability.

rs-fMRI data acquisition

The rs-fMRI scan was performed on a $3 \mathrm{~T}$ scanner (Siemens Magnetom Trio, A Tim System) with a 12-channel phasedarray head coil at BNU Imaging Center for Brain Research, Beijing, China. During rs-fMRI scanning, participants were instructed to close their eyes, keep still, and not think about anything systematically or fall asleep. The resting state scanning consisted of 240 contiguous EPI volumes (TR = $2000 \mathrm{~ms} ; \mathrm{TE}=30 \mathrm{~ms}$; flip angle $=90^{\circ}$; number of slices $=$ 33 ; matrix $=64 \times 64 ; \mathrm{FOV}=200 \times 200 \mathrm{~mm}^{2}$; acquisition voxel size $=3.1 \times 3.1 \times 3.6 \mathrm{~mm}^{3}$ ). Moreover, high-resolution T1-weighted images were also acquired with magnetization prepared rapid gradient echo sequence (MPRAGE: TR/TE/TI $=2530 / 3.39 / 1100 \mathrm{~ms}$; flip angle $=7^{\circ}$; matrix $=256 \times 256$ ) for spatial registration. 128 contiguous sagittal slices were obtained with $1 \times 1 \mathrm{~mm}^{2}$ in-plane resolution and $1.33 \mathrm{~mm}$ slice thickness.

\section{Data preprocessing}

Preprocessing was performed using FSL (www.fmrib.ox.ac. $\mathrm{uk} / \mathrm{fs} 1 /$ ). For the rs-fMRI data, the first four volumes were discarded for signal equilibrium. Preprocessing steps of rsfMRI data consisted of spatial Gaussian smoothing (FWHM = $6 \mathrm{~mm}$ ), realignment, motion correction (by aligning each volume to the middle volume of the image with MCFLIRT), intensity normalization, and removing linear trends. Five participants whose head motion was greater than $2.0 \mathrm{~mm}$ displacement in any of the $\mathrm{x}, \mathrm{y}$, or $\mathrm{z}$ directions or $2.0^{\circ}$ of any angular motion throughout the rs-fMRI scan were excluded from further analyses. We also computed mean framewise displacement (FD) (Van Dijk, Sabuncu, \& Buckner, 2012) and found no participant with a mean FD $>0.25 \mathrm{~mm}$. Although there was no significant correlation between FD and EWB $(r=-0.06, p=0.356)$, we also used the mean FD of each participant as a nuisance covariate in group-level analyses.

Registration of each participant's high-resolution anatomical image to a common stereotaxic space (the Montreal Neurological Institute 152-brain template with a resolution of $2 \times 2 \times 2 \mathrm{~mm}^{3}$; MNI152) was then accomplished using a two-step process (Andersson, Jenkinson, \& Smith, 2007). A 12-degrees-of-freedom linear affine was first carried out with FLIRT (Jenkinson \& Smith, 2001; Jenkinson, Bannister, Brady, \& Smith, 2002) and then the registration was refined with FNIRT nonlinear registration (Andersson et al., 2007). Registration of each participant's functional images to the high-resolution anatomical images was performed with FLIRT to produce a 6-degrees-of-freedom affine transformation matrix.

\section{Calculation of fALFF}

Because LFF is sensitive to signals in the gray matter (Biswal et al., 1995), additional analyses were conducted on a gray matter mask. For a time series in each voxel, the sum of amplitudes within a low frequency range $(0.01-0.1 \mathrm{~Hz})$ was extracted. The fALFF was then computed as the fractional 
sum of amplitudes within the low frequency range that was divided by the sum of amplitude across the entire frequency range $(0-0.25 \mathrm{~Hz}$ ) (Zuo et al., 2010). As a normalized index of ALFF, fALFF is less susceptible to artifactual signals in regions located within the vicinity of vessels and/or significant pulsatile motion (Zuo et al., 2010; Zou et al., 2008). To reduce the global effects of variability across participants, the fALFF of each voxel was divided by the mean global fALFF value within the gray matter mask for each participant (Dai et al., 2012; Wang, Han, He, Caramazza, Song, \& Bi, 2013). The fALFF maps were then registered to MNI152 space by applying the previously calculated transformation information.

\section{fALFF-behavior correlation analysis}

To detect the brain regions for which AALF values predicted EWB, we conducted a whole-brain partial correlation analysis using the general linear model, with age, gender, and mean FD as the confounding covariates, and the EWB score as the covariates of interest. Multiple comparison correction was performed using the 3dClustSim program in AFNI (http:// afni.nimh.nih.gov/pub/dist/doc/program_help/3dClustSim. $\mathrm{html}$ ). We set a corrected cluster threshold of $p<0.01$ (single voxel $p<0.005$, cluster size $\geq 66$ voxels; $528 \mathrm{~mm}^{3}$ ).

\section{Connectivity-behavior correlation analysis}

To explore whether the key region(s) we found in the fALFFbehavior analysis interact with other brain regions or among themselves to form a functional network for EWB, we computed the resting-state functional connectivity between the core region(s) identified to be linked with the EWB score and all other voxels in the brain. We created spherical seed regions of interest (ROIs; radius $=5 \mathrm{~mm}$ ) according to the coordinates of peaks in the significant region(s) observed in the fALFF-behavior correlation analysis. This method has been widely used to construct RSFC networks (Cox et al., 2012; Li et al., 2014; Wang et al., 2013; Wei et al., 2012; Xu et al., 2014).To examine the network's functional relevance for EWB, we then correlated the RSFC strength with the EWB score. Before the RSFC analysis, a temporal band-pass filter (0.01-0.1 Hz) was applied with FSLMATHS to reduce lowfrequency drifts and high-frequency noise. Moreover, six head motion parameters, mean global signal, white matter signal, cerebrospinal fluid signal, and the derivatives of each of these signals were regressed out from BOLD signals in FSL. The RSFC analysis was performed in FSL. The four-dimensional residual time series were transformed into MNI152 standard space. For each participant, mean time series of the seed $\mathrm{ROI}(\mathrm{s})$ were calculated and then correlated with time series of all other voxels in the brain. The correlation coefficients were transformed to Fisher z-scores to form a z-functional connectivity (zFC) map for each participant. We then performed the zFC-behavior partial correlation analysis, with participants' age, gender, and mean FD removed. The threshold of significance was determined by running $3 \mathrm{dClustSim}$ in the previous mask. A corrected cluster threshold was set as $p$ $<0.01$ (single voxel $p<0.005$, cluster size $\geq 66$ voxels; $528 \mathrm{~mm}^{3}$ ).

\section{Multiple mediation analysis}

In order to examine how personality traits are differently associated with spontaneous brain activities in relation to EWB, we conducted a multiple mediation analysis, using the SPSS macro programmed by Preacher and Hayes (2008). Compared to simple mediation analysis, multiple mediation analysis may determine which mediator variable plays a more important role in the associations via the methods comparing specific indirect effects. Multiple mediation analyses employed a bootstrapping approach to test the significance of the indirect effect of the independent variable (IV; personality traits) on the outcome variable (DV; EWB) through the mediator (M; fALFF of brain regions), which was calculated as the product of the effect of IV on $\mathrm{M}$ and that of $\mathrm{M}$ on DV. The total effect (Path c) refers to the relationship between IV and DV without controlling for M. The direct effect (Path c') refers to the relationship between IV and DV after controlling for M. An estimate of the specific indirect effect was derived from the mean of 5,000 bootstrap samples, and bias corrected and accelerated $95 \%$ confidence intervals (CIs) were calculated (Preacher \& Hayes, 2008). If an empirical $95 \% \mathrm{CI}$ did not include zero, the indirect effect was significant at the 0.05 level.

\section{Results}

Identification of the neural correlates of EWB

At the regional level, we correlated participants' EWBs with the fALFF values for each voxel across the whole brain. We found that after controlling for age, gender, and mean FD, EWB was positively correlated with the fALFF in two clusters: one in the right posterior superior temporal gyrus (pSTG, MNI: 66, -20, 6; z $=4.30$; Cluster size $=1192 ; p<0.01)($ Fig. $1 \mathrm{~A}$ and B, Table 1) and the other that included the left thalamus and extended to the right thalamus (MNI: $-6,-16,0 ; \mathrm{z}=3.94$; Cluster size $=800 ; p$ $<0.01$ ) (Fig. $1 \mathrm{C}$ and D, Table 1). No significant negative correlations were identified in this analysis.

At the functional connectivity level, we computed the RSFC between each of the regions identified to be associated with EWB (seed ROIs) and all other voxels in the brain, and then correlated the RSFC strength with the EWB score. We found that after controlling for age, gender, and mean FD for 


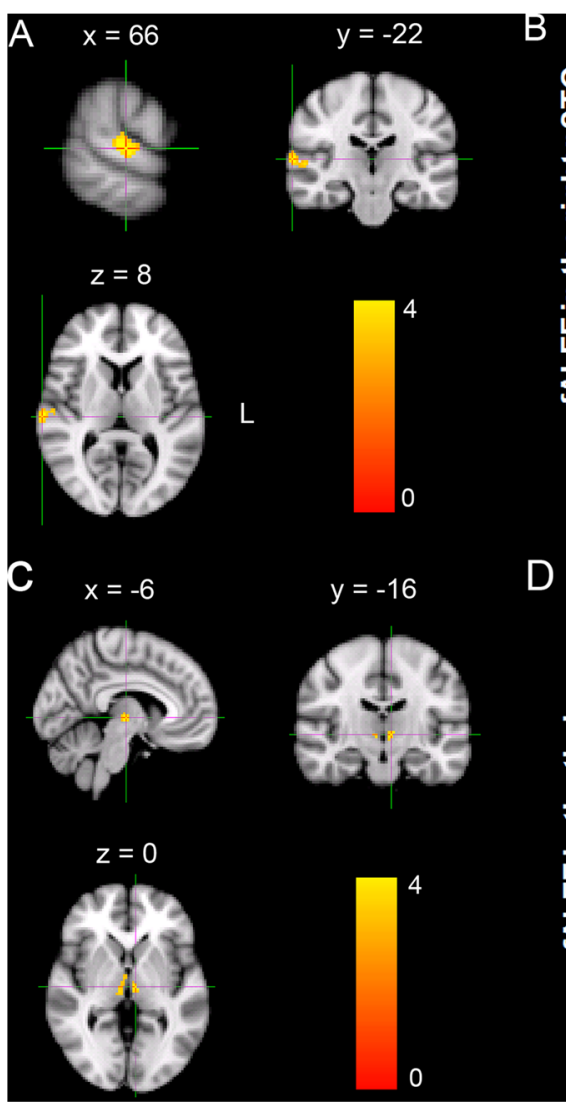

Fig. 1 Brain regions that are correlated with eudaimonic well-being. (A) The fractional amplitude of low-frequency fluctuations (fALFF) in the right posterior superior temporal gyrus (pSTG) was positively correlated with eudaimonic well-being (EWB). The coordinate is shown in the Montreal Neurological Institute (MNI) stereotactic space. (B) Scatter

the thalamus seed ROI, EWB was negatively correlated with RSFC strength between the thalamus (the seed ROI) and the right insula (MNI: 36, 14, $-2 ; 720 \mathrm{~mm}^{3}$; cluster-corrected $p$ $<0.01$ ) (Fig. 2, Table 2). Specifically, individuals with high EWB scores exhibited weaker positive RSFC between these two regions. No significant results were identified for the right pSTG seed ROI in this analysis.

Previous studies have revealed that mean global signal can reduce non-neural signal correlations or lead to spurious anticorrelations (Murphy, Birn, Handwerker, Jones, \&

Table 1 Regions correlating with eudaimonic well-being

\begin{tabular}{|c|c|c|c|c|c|c|}
\hline \multirow{2}{*}{\multicolumn{2}{|c|}{$\begin{array}{l}\text { Region Side } \\
\text { Positive correlation }\end{array}$}} & \multicolumn{3}{|c|}{ MNI coordinate $x y z$} & \multirow[t]{2}{*}{$Z$} & \multirow[t]{2}{*}{ Cluster size $\left(\mathrm{mm}^{3}\right)$} \\
\hline & & & & & & \\
\hline pSTG & $\mathrm{R}$ & 66 & -22 & 8 & 4.30 & $1192 *$ \\
\hline Thalamus & $\mathrm{L}$ & -6 & -16 & 0 & 3.94 & $800^{*}$ \\
\hline
\end{tabular}

$\mathrm{MNI}=$ Montreal Neurological Institute; $\mathrm{L}=$ left; $\mathrm{R}=$ right; $\mathrm{pSTG}=$ posterior superior temporal gyrus. All z-scores reflect a threshold of $p$ $<0.005$ (uncorrected). $* p<0.01$ corrected for multiple comparisons at the cluster level

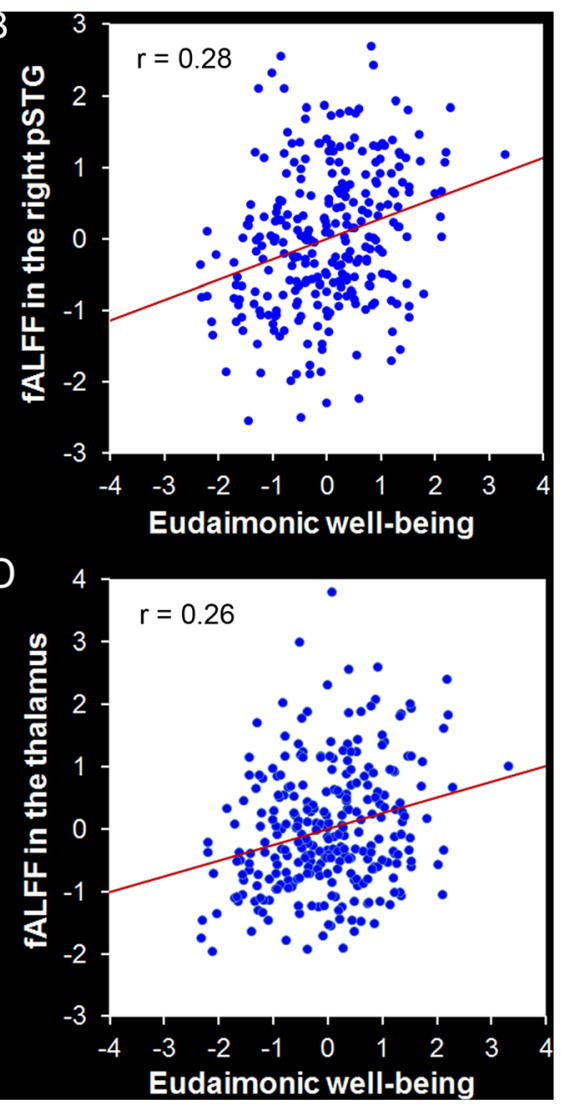

plots depicting correlations between fALFF in the right pSTG and individual differences in EWB. (C) The fALFF in the thalamus was positively correlated with EWB. The coordinate is shown in the MNI stereotactic space. (D) Scatter plots depicting correlations between fALFF in the thalamus and individual differences in EWB

Bandettini, 2009; Saad et al., 2012). Thus we retested the correlation between RSFC and EWB when the mean global signal was not regressed out in the preprocessing analysis. We found that, after controlling for age, gender, and mean FD for the thalamus seed ROI, the negative correlation of EWB with RSFC strength between the thalamus and the right insula remained (MNI: 36, 14, $-2 ; 800 \mathrm{~mm} 3 ; \mathrm{Z}=3.38$; clustercorrected $\mathrm{p}<0.01)$. Although the size of the cluster exhibited small changes, the significant region was identical to those identified in initial analyses.

In order to assess the joint contributions of intrinsic brain activities in predicting EWB, we carried out a multiple linear regression. The dependent variable was participants' EWB scores and the independent variables were the fALFF in the pSTG and thalamus and the thalamic-insular connectivity. The results revealed that these independent variables explained $12.3 \%$ of the variance in $\mathrm{EWB}\left(\mathrm{R}^{2}=0.123 ; \mathrm{F}_{[3,268]}\right.$ $=12.56 ; p<0.001)$ and all regression coefficients for the all brain regions $(\beta=.16,-.20, p s<0.01)$ were significant. These results suggest that the fALFF in the pSTG and thalamus and the connectivity between the thalamus and insula may explain the unique variance in individual differences in EWB. 


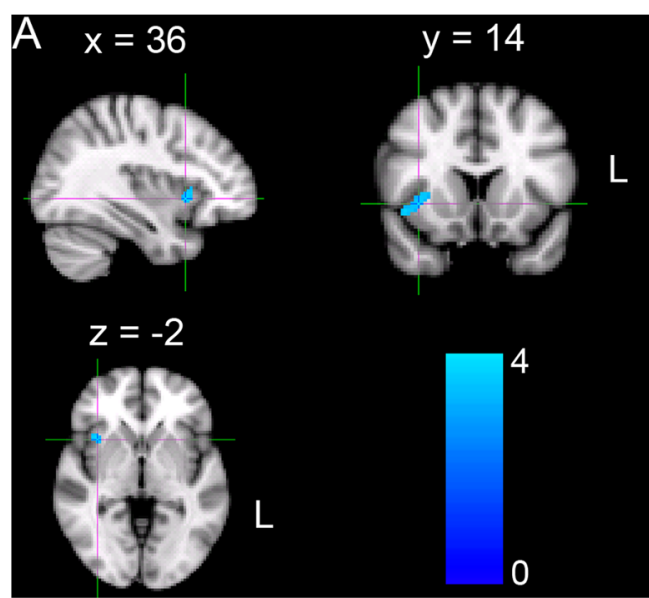

Fig. 2 Functional connectivity that correlated with eudaimonic wellbeing. (A) The strength of the thalamus and the right insula was negatively correlated with eudaimonic well-being (EWB). The coordinate is shown in the Montreal Neurological Institute (MNI)

The association between EWB and personality traits is meditated by different neural substrates

We collected the measures of the "big five" personality traits from a subset $(\mathrm{n}=265)$ of the participants studied in the fALFF-behavior correlation analysis. Consistent with previous studies (Abbott et al., 2008; Augusto Landa et al., 2010; Garcia, 2011; Grant et al., 2009), neuroticism $(r=-0.52, p$ $<0.001$; FDR corrected), extraversion $(r=0.48, p<0.001$; FDR corrected), and conscientiousness $(\mathrm{r}=0.51, p<0.001)$ were highly correlated with EWB, whereas openness $(r=$ $0.30, p<0.001$; FDR corrected) and agreeableness $(r=0.14$, $p=0.015$; FDR corrected) were moderately correlated with EWB. Furthermore, a multiple regression analysis revealed that they jointly explained $47 \%$ of the variance in EWB.

To test how personality traits differently influence intrinsic brain activities associated with EWB, we conducted a correlation analysis between personality and EWB-related regions and connectivity. We found that neuroticism was correlated with the fALFF in the pSTG $(\mathrm{r}=-0.15, p=0.048$; FDR corrected $)$ and thalamus $(\mathrm{r}=-0.20, p=0.005$; FDR corrected $)$ and the thalamicinsular connectivity ( $\mathrm{r}=0.17, p=0.021$; FDR corrected). Extraversion was correlated with the fALFF in the pSTG $(\mathrm{r}=$ $0.21, p=0.005$; FDR corrected) and thalamus $(\mathrm{r}=0.19, p=$

Table 2 Functional connectivity correlating with eudaimonic wellbeing

\begin{tabular}{lllll}
\hline Region & Side & MNI coordinate $x y z$ & $Z$ & Cluster size $\left(\mathrm{mm}^{3}\right)$
\end{tabular}

Negative correlation

$\begin{array}{lllllll}\text { Insula } & R & 36 & 14 & -2 & 3.26 & 720 *\end{array}$

$\mathrm{MNI}=$ Montreal Neurological Institute; $\mathrm{L}=$ left; $\mathrm{R}=$ right. All z-scores reflect a threshold of $p<0.005$ (uncorrected). ${ }^{*} p<0.01$ corrected for multiple comparisons at the cluster level

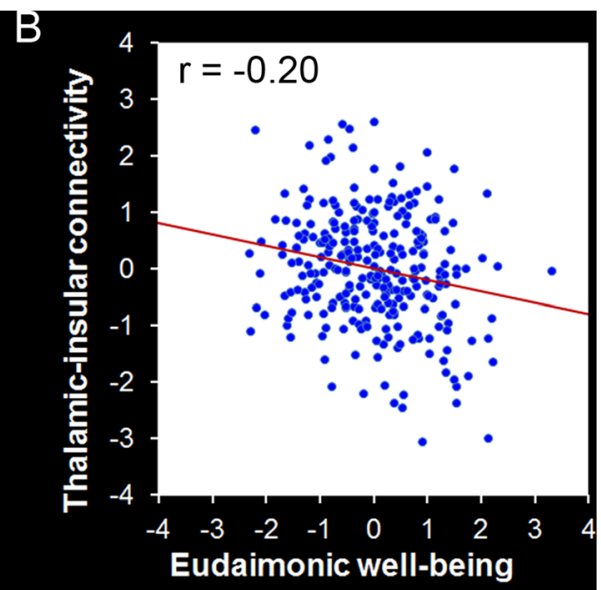

stereotactic space. (B) Scatter plots depicting correlations between the strength of thalamic-insular connectivity and individual differences in EWB

0.0075; FDR corrected). Conscientiousness was correlated with the fALFF in the thalamus ( $\mathrm{r}=0.21, p=0.005$; FDR corrected $)$. No other personality factors showed a significant correlation with these clusters. This indicates that personality traits are differentially associated with EWB-related spontaneous brain activities. Next, to test whether the associations between EWB and personality traits are meditated by distinct neural substrates, we carried out three multiple mediation analyses for the three personality traits involved.

For neuroticism, after regressing out the right $\mathrm{pSTG}$, thalamus, and thalamic-insular connectivity, the effect of neuroticism on EWB was reduced, though still significant (Fig. 3). A bootstrap simulation $(n=5000)$ was further used to test the significance of the specific indirect effects through these factors. We found that the pSTG, thalamus, and thalamic-insular connectivity significantly mediated the association between neuroticism and EWB $(p<0.05)$. Further indirect effect contrasts demonstrated that the indirect effect size for three mediators had no statistically significant differences, indicating that they played equally important roles in the association between neuroticism and EWB (Table 3).

For extraversion, after regressing out the right $\mathrm{pSTG}$ and thalamus, the effect of extraversion on EWB was reduced, though still significant (Fig. 3). The bootstrap simulation indicated that only the pSTG and thalamus significantly mediated the association between extraversion and EWB $(p<0.05)$. Further indirect effect contrasts revealed that the indirect effect size for two mediators had no statistically significant differences, indicating that they played equally important roles in the association between extraversion and EWB (Table 3).

For conscientiousness, after regressing out the right pSTG, the effect of conscientiousness on EWB was reduced, though still significant (Fig. 3). The bootstrap simulation indicated that the thalamus significantly mediated the association between conscientiousness and EWB $(p<0.05)$ (Table 3$)$. 


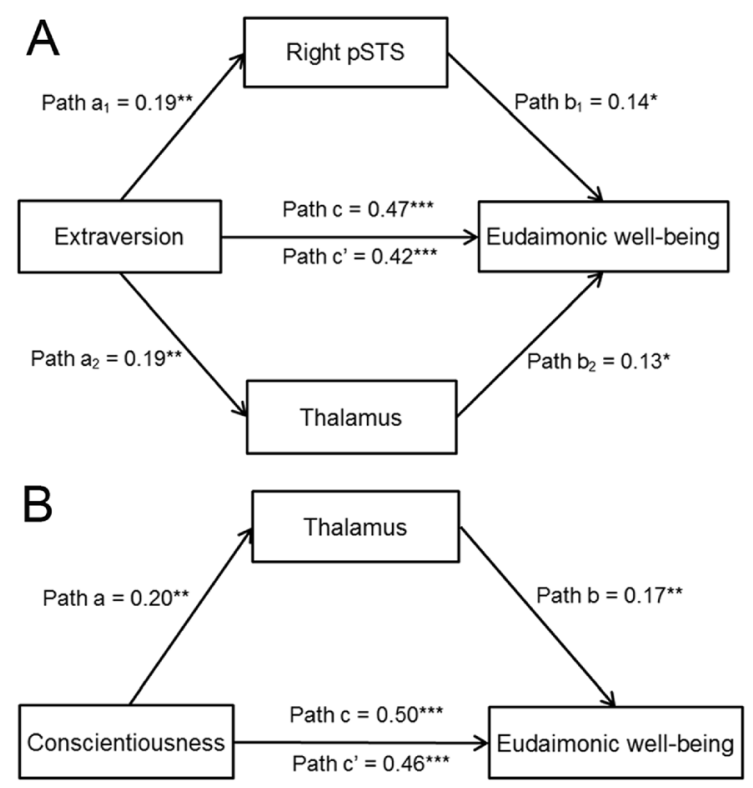

Fig. 3 The pSTG, thalamus, and thalamic-insular connectivity mediate the impact of on personality on eudaimonic well-being. Depicted is the path diagram (including standard regression coefficients) of the multiple mediation analysis demonstrating that personality affects individuals'

In addition, we used the same mediation analysis procedures to examine the mediational models in which intrinsic activities in relevant brain regions could mediate the relationship between EWB and personality traits. The results showed that the empirical $95 \%$ CI of all three indirect effects included zero, which indicated intrinsic activities in these brain regions did not mediate the relationship between EWB and personality traits. Therefore, we concluded that intrinsic activities in these brain regions mediated the association between personality traits and EWB.

Table 3 Unstandardized indirect effects and $95 \%$ confidence intervals (CIs)

\begin{tabular}{|c|c|c|c|}
\hline \multirow[t]{2}{*}{ Model pathways } & \multirow[t]{2}{*}{ Estimated } & \multicolumn{2}{|c|}{$95 \% \mathrm{CI}$} \\
\hline & & Lower & Upper \\
\hline Neuroticism $\rightarrow$ pSTG $\rightarrow$ EWB & $-0.48^{\mathrm{a}}$ & -1.16 & -0.09 \\
\hline Neuroticism $\rightarrow$ Thalamus $\rightarrow$ EWB & $-0.42^{\mathrm{a}}$ & -1.06 & -0.05 \\
\hline $\begin{array}{l}\text { Neuroticism } \rightarrow \text { Thalamic-insular } \\
\text { connectivity } \rightarrow \text { EWB }\end{array}$ & $-0.36^{\mathrm{a}}$ & -0.98 & -0.05 \\
\hline IndEff $_{(\mathrm{pSTG})}$ minus IndEff (Thalamus) & -0.06 & -0.85 & 0.59 \\
\hline $\begin{array}{l}\text { IndEff }_{(\mathrm{pSTG})} \text { minus IndEff } \\
\text { connectivity) }\end{array}$ & -0.11 & -0.82 & 0.57 \\
\hline $\begin{array}{l}\text { IndEff (Thalamus) minus IndEff (Thalamic- }_{\text {(insular connectivity) }} \\
\text { ing }\end{array}$ & -0.05 & -0.69 & 0.62 \\
\hline Extraversion $\rightarrow$ pSTG $\rightarrow$ EWB & $0.53^{\mathrm{a}}$ & 0.12 & 1.21 \\
\hline Extraversion $\rightarrow$ Thalamus $\rightarrow$ EWB & $0.47^{\mathrm{a}}$ & 0.10 & 1.17 \\
\hline IndEff $_{(\mathrm{pSTG})}$ minus IndEff (Thalamus) & 0.06 & -0.76 & 0.83 \\
\hline Conscientiousness $\rightarrow$ Thalamus $\rightarrow$ EWB & $0.67^{\mathrm{a}}$ & 0.21 & 1.45 \\
\hline
\end{tabular}

${ }^{\mathrm{a}}$ Empirical 95 \% CI does not overlap with zero

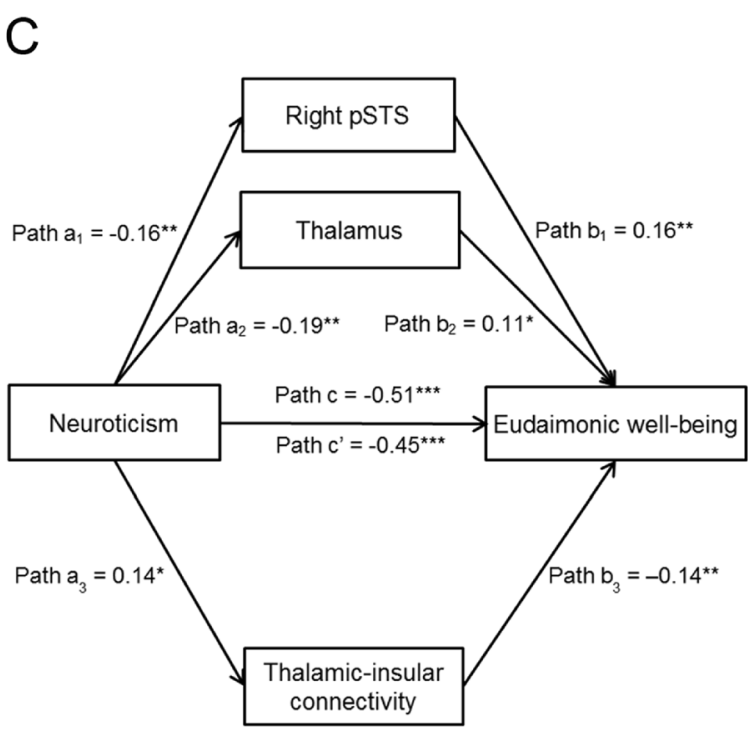

eudaimonic well-being through intrinsic brain activity in these regions. Path a, path $b$, and path $\mathrm{c}$ are significant, and the specific indirect effects are significant. $*$ : $\mathrm{p}<0.05 .{ }^{*}: \mathrm{p}<0.01 .{ }^{* * *}: \mathrm{p}<0.001$

\section{Discussion}

The purpose of the present study was to test how basic personality traits are differently associated with EWB of healthy individuals within a neuroscientific framework. Behaviorally, we found that extraversion, neuroticism, and conscientiousness were more strongly related to EWB than agreeableness and openness, and accounted for approximately half of the variance in EWB; this finding is consistent with that of previous studies (Abbott et al., 2008; Augusto Landa et al., 2010; Garcia, 2011; Grant et al., 2009). Neurally, we demonstrated that EWB was positively correlated with the fALFF in the right pSTG and thalamus and negatively correlated with the strength of the thalamic-insular functional connectivity. Crucially, we found the distinct contributions of specific personality traits to predict spontaneous brain activity associated with EWB. Specifically, at the regional level, the pSTG and thalamus mediated the effect of neuroticism on EWB as well as the effect of extraversion on EWB, whereas the thalamus mediated the effect of conscientiousness on EWB. At the functional connectivity level, the thalamicinsular connectivity only mediated the effect of neuroticism on EWB. Taken together, we provide one of the first examples of rs-fMRI evidence that decouples the contribution of different personality traits to EWB.

At the regional level, we found that the AALFF of the right pSTG and thalamus neuroticism mediated the association between neuroticism and EWB. This is consistent with the findings of Luo et al. (2014), who reported that happiness was correlated with the regional homogeneity of intrinsic brain 
activity in the thalamus and superior temporal lobe. Furthermore, previous imaging studies have shown that neuroticism predicts structural variations and activity in the STG and thalamus in response to negative stimuli (DeYoung, Hirsh, Shane, Papademetris, Rajeevan, \& Gray, 2010; Kumari, Das, Wilson, Goswami, \& Sharma, 2007; Omura, Constable, \& Canli, 2005; Servaas et al, 2013). The STG has been implicated in several types of self-referential processes such as self-recognition (Kircher et al., 2001; Platek \& Kemp, 2009) and autobiographical memory (Spreng \& Grady, 2010). The thalamus plays a crucial role in sleep regulation (Sherman \& Guillery, 2006), self-consciousness (Crick \& Koch, 2003; Damasio et al., 2000; Tsakiris, Hesse, Boy, Haggard, \& Fink, 2007), self-recognition (Devue \& Brédart, 2011; Northoff \& Panksepp, 2008), and meditation (Christoff, Gordon, Smallwood, Smith, \& Schooler, 2009; Zeidan, Martucci, Kraft, Gordon, McHaffie, \& Coghill, 2011). All these processes closely reflect the multifaceted description of EWB as reflected in high levels of self-awareness, self-knowledge, and self-reflection in pursuit of intrinsic goals (Ryan, Huta, \& Deci, 2008; Ryff \& Keyes 1995). Thus, spontaneous activities in the pSTG and thalamus were associated with neuroticism and EWB, probably through self-related processes such as self-recognition, self-consciousness, and autobiographical memory.

More importantly, at the functional connectivity level, we found that the thalamic-insular functional connectivity mediated the association between neuroticism and EWB. On the one hand, this is the first study, as far as we know, showing the relationship between EWB and thalamic-insular connectivity. This observation is in line with previous studies reporting the role of insula in EWB (Lewis et al. 2013), and a stronger thalamus-insular structural and functional connectivity at rest in individuals with schizophrenia (Corradi-Dell'Acqua et al., 2012; Klingner et al., 2013), who report lower EWB than healthy individuals (Strauss, Sandt, Catalano, \& Allen, 2012). On the other hand, this result suggests that the thalami-insular connectivity may be an important neural mechanism underlying neuroticism. Task-based fMRI studies have shown that neuroticism predicts activity in the right insula during risky or certain decision-making (Feinstein, Stein, \& Paulus, 2006; Paulus, Rogalsky, Simmons, Feinstein, \& Stein, 2003). In neurotic individuals, the stronger thalami-insular connectivity seems to support Sylvester et al.'s (2012) hypothesis that an overactive insular network is an endophenotype of anxiety disorders. The insula has been stated to play a crucial role in interoception, awareness of sensations and movements, self-recognition, emotional awareness, cognitive control, and performance monitoring (Craig, 2009; Singer, Critchley, \& Preuschoff, 2009). All these functions seem to be essential for the facets of neuroticism, including poor self-consciousness, poor impulse control, hostility, anxiety, depression, and worry (Costa \& McCrae, 1992). In sum, neuroticism might be associated with EWB through a set of capacities including self-consciousness, emotional awareness, cognitive control, and performance monitoring, thus leading to the engagement of the thalamic-insular connectivity.

In contrast, only regional spontaneous activity in the pSTG and thalamus accounted for the association between extraversion and EWB. Previous studies have shown that extraversion is correlated with the rs-fMRI signal in the STG (Kumari, Williams, \& Gray, 2004; Wei et al, 2014) and the thalamus (Kumari et al., 2004). Moreover, task-based fMRI studies have demonstrated that extraversion predicts the STG activity during humor appreciation (Mobbs, Hagan, Azim, Menon, \& Reiss, 2005) and the thalamus activity in response to happy faces (Suslow et al., 2010). In addition to the role of the STG and thalamus in self-related processes, the STG is also implicated in socially related processes such as are involved in voice perception (Belin, Fecteau, \& Bédard, 2004), social perception (Blakemore \& Decety, 2001; Zilbovicius, Meresse, Chabane, Brunelle, Samson, \& Boddaert, 2006), and theory of mind (Spreng \& Grady, 2010). These two types of processes are crucial to social functioning, fore example in interpersonal communication. This is consistent with the description of extraversion in Costa and McCrae's FFM model, according to which the core feature of extraversion is thought to be the disposition to engage in social behavior. Thus, it is plausible that spontaneous activities in the pSTG and thalamus support the association between extraversion and EWB.

Finally, we found that, in contrast to neuroticism and extraversion, only the fALFF in the thalamus supported the association between conscientiousness and EWB. Relative to neuroticism and extraversion, little neuroimaging data are available regarding the neural correlates of conscientiousness. Our study indicated that the thalamus might play an important role in conscientiousness. Previous studies have explored the neural correlates of persistence, which is highly correlated with conscientiousness and conceived as a facet of conscientiousness, and found that persistence is associated with a decreased glucose metabolic rate in the thalamus (Haier, Sokolski, Katz, \& Buchsbaum, 1987). Given the role of the thalamus in self-regulation processes, individual differences in the thalamus may serve as one mechanism through which conscientious individuals who engage in goal-directed activity and exert control over oneself and one's environment possess higher levels of EWB.

Several limitations of the present study should be mentioned. First, the data relied on self-report measures. Although they were selected for their good reliability and validity, selfreport measures are vulnerable to bias, such as social desirability. The use of multiple methods for evaluation (e.g., peer rating) may lower the impact of subjectivity. Second, the present study was cross-sectional, which precludes the drawing of conclusions concerning the causal nature of emerging relationships. Further studies are needed to investigate the longitudinal relationship between well-being, personality, 
and intrinsic brain activities. Finally, although our study used a data-driven method to define EWB-related ROIs for subsequent seed-based RSFC analyses (see also Cox et al., 2012; Li et al., 2014; Xu et al., 2014; Wang et al., 2013; Wei et al., 2012), future studies should also consider using other well established methods (e.g., ICA templates, standard seeds, reference to pre-existing node sets) to explore the EWBrelated brain network.

\section{Conclusions}

In summary, we employed the fALFF method to investigate the different effects of basic personality traits on well-being in healthy adults. The present findings indicate that: (1) The fALFF values of the pSTG and thalamus could be used to predict individual differences in EWB. (2) The connectivity between the thalamus and insula further confirms the roles of these regions in EWB and reveals a potential new mechanism for EWB (i.e., through resting-state fluctuations). (3) Although all the five personality traits, especially neuroticism, extraversion, and conscientiousness contribute to EWB, they may be associated with EWB through different intrinsic brain activity patterns. Finally, our findings have important clinical implications, providing potential biomarkers for the early detection of psychological functioning deterioration. Furthermore, strength-training interventions (especially those related to emotional stability, extraversion, and conscientiousness) can be used to improve health and well-being.

Acknowledgments This study was funded by the National Natural Science Foundation of China (31230031, 91132703, 31221003), the National Basic Research Program of China (2014CB846103), National Social Science Foundation of China (11\&ZD187), and Changjiang Scholars Programme of China.

Conflict of interest The authors declare no competing interests.

\section{References}

Abbott, R. A., Croudace, T. J., Ploubidis, G. B., Kuh, D., Richards, M., \& Huppert, F. A. (2008). The relationship between early personality and midlife psychological well-being: Evidence from a UK birth cohort study. Social Psychiatry and Psychiatric Epidemiology, 43(9), 679-687.

Andersson, J. L., Jenkinson, M., \& Smith, S. (2007). Non-linear registration, aka Spatial normalisation. FMRIB Analysis Group Technical Reports.

Augusto Landa, J. M., Martos, M. P., \& Lopez-Zafra, E. (2010). Emotional intelligence and personality traits as predictors of psychological well-being in Spanish undergraduates. Social Behavior and Personality, 38(6), 783-793.

Belin, P., Fecteau, S., \& Bédard, C. (2004). Thinking the voice: Neural correlates of voice perception. Trends in Cognitive Sciences, 8(3), $129-135$.
Biswal, B. B. (2012). Resting state fMRI: A personal history. NeuroImage, 62(2), 938-944.

Biswal, B., Zerrin Yetkin, F., Haughton, V. M., \& Hyde, J. S. (1995). Functional connectivity in the motor cortex of resting human brain using echo-planar MRI. Magnetic Resonance in Medicine, 34(4), 537-541.

Blakemore, S.-J., \& Decety, J. (2001). From the perception of action to the understanding of intention. Nature Reviews Neuroscience, 2(8), $561-567$.

Christoff, K., Gordon, A. M., Smallwood, J., Smith, R., \& Schooler, J. W. (2009). Experience sampling during fMRI reveals default network and executive system contributions to mind wandering. Proceedings of the National Academy of Sciences, 106(21), 8719-8724.

Corradi-Dell'Acqua, C., Tomelleri, L., Bellani, M., Rambaldelli, G., Cerini, R., Pozzi-Mucelli, R., et al. (2012). Thalamic-insular dysconnectivity in schizophrenia: Evidence from structural equation modeling. Human Brain Mapping, 33(3), 740-752.

Costa, P. T., Jr., \& McCrae, R. R. (1990). Personality disorders and the five-factor model of personality. Journal of Personality Disorders, 4(4), 362-371.

Costa, P. T., \& McCrae, R. R. (1992). Professional manual: Revised NEO personality inventory (NEO-PI-R) and NEO five-factor inventory (NEO-FFI). Odessa, FL: Psychological Assessment Resources.

Costa, P. T., Jr., \& McCrae, R. R. (1995). Domains and facets: Hierarchical personality assessment using the Revised NEO Personality Inventory. Journal of Personality Assessment, 64(1), 21-50.

Cox, C. L., Uddin, L. Q., Di Martino, A., Castellanos, F. X., Milham, M. P., \& Kelly, C. (2012). The balance between feeling and knowing: Affective and cognitive empathy are reflected in the brain's intrinsic functional dynamics. Social Cognitive and Affective Neuroscience, 7(6), 727-737.

Craig, A. (2009). How do you feel-now? The anterior insula and human awareness. Nature Reviews Neuroscience, 10(1), 59-70.

Crick, F., \& Koch, C. (2003). A framework for consciousness. Nature Neuroscience, 6(2), 119-126.

Dai, Z., Yan, C., Wang, Z., Wang, J., Xia, M., Li, K., et al. (2012). Discriminative analysis of early Alzheimer's disease using multimodal imaging and multi-level characterization with multi-classifier (M3). Neurolmage, 59(3), 2187-2195.

Damasio, A. R., Grabowski, T. J., Bechara, A., Damasio, H., Ponto, L. L., Parvizi, J., et al. (2000). Subcortical and cortical brain activity during the feeling of self-generated emotions. Nature Neuroscience, 3(10), 1049-1056.

Devue, C., \& Brédart, S. (2011). The neural correlates of visual selfrecognition. Consciousness and Cognition, 20(1), 40-51.

DeYoung, C. G., Hirsh, J. B., Shane, M. S., Papademetris, X., Rajeevan, N., \& Gray, J. R. (2010). Testing predictions from personality neuroscience brain structure and the big five. Psychological Science, 21(6), 820-828.

Feinstein, J. S., Stein, M. B., \& Paulus, M. P. (2006). Anterior insula reactivity during certain decisions is associated with neuroticism. Social Cognitive and Affective Neuroscience, 1(2), 136-142.

Fox, M. D., \& Raichle, M. E. (2007). Spontaneous fluctuations in brain activity observed with functional magnetic resonance imaging. Nature Reviews Neuroscience, 8(9), 700-711.

Gallagher, M. W., Lopez, S. J., \& Preacher, K. J. (2009). The hierarchical structure of well-being. Journal of Personality, 77(4), 1025-1050.

Garcia, D. (2011). Two models of personality and well-being among adolescents. Personality and Individual Differences, 50(8), 12081212.

Grant, S., Langan-Fox, J., \& Anglim, J. (2009). The big five traits as predictors of subjective and psychological well-being. Psychological Reports, 105(1), 205-231.

Haier, R. J., Sokolski, K., Katz, M., \& Buchsbaum, M. S. (1987). The study of personality with positron emission tomography. Personality Dimensions and Arousal (pp. 251-267): Springer. 
Han, Y., Wang, J., Zhao, Z., Min, B., Lu, J., Li, K., et al. (2011). Frequency-dependent changes in the amplitude of low-frequency fluctuations in amnestic mild cognitive impairment: A resting-state fMRI study. NeuroImage, 55(1), 287-295.

Headey, B., \& Wearing, A. (1989). Personality, life events, and subjective well-being: Toward a dynamic equilibrium model. Journal of Personality and Social Psychology, 57(4), 731-739.

Heller, A. S., van Reekum, C. M., Schaefer, S. M., Lapate, R. C., Radler, B. T., Ryff, C. D., et al. (2013). Sustained striatal activity predicts eudaimonic well-being and cortisol output. Psychological Science, 24(11), 2191-2200.

Hoptman, M. J., Zuo, X.-N., Butler, P. D., Javitt, D. C., D'Angelo, D., Mauro, C. J., et al. (2010). Amplitude of low-frequency oscillations in schizophrenia: A resting state fMRI study. Schizophrenia Research, 117(1), 13-20.

Jenkinson, M., Bannister, P., Brady, M., \& Smith, S. (2002). Improved optimization for the robust and accurate linear registration and motion correction of brain images. NeuroImage, 17(2), 825-841.

Jenkinson, M., \& Smith, S. (2001). A global optimisation method for robust affine registration of brain images. Medical Image Analysis, $5(2), 143-156$

Jiao, Q., Ding, J., Lu, G., Su, L., Zhang, Z., Wang, Z., et al. (2011). Increased activity imbalance in fronto-subcortical circuits in adolescents with major depression. PloS One, 6(9), e25159.

Kircher, T. T., Senior, C., Phillips, M. L., Rabe-Hesketh, S., Benson, P. J., Bullmore, E. T., et al. (2001). Recognizing one's own face. Cognition, 78(1), B1-B15.

Klingner, C. M., Langbein, K., Dietzek, M., Smesny, S., Witte, O. W., Sauer, H., et al. (2013). Thalamocortical connectivity during resting state in schizophrenia. European Archives of Psychiatry and Clinical Neuroscience, 1-9.

Kumari, V., Das, M., Wilson, G. D., Goswami, S., \& Sharma, T. (2007). Neuroticism and brain responses to anticipatory fear. Behavioral Neuroscience, 121(4), 643-652.

Kumari, V., Williams, S. C., \& Gray, J. A. (2004). Personality predicts brain responses to cognitive demands. Journal of Neuroscience, 24(47), 10636-10641

Lewis, G. J., Kanai, R., Rees, G., \& Bates, T. C. (2013). Neural correlates of the "good life": Eudaimonic well-being is associated with insular cortex volume. Social Cognitive and Affective Neuroscience. doi:10. $1093 /$ scan/nst032

Li, F., He, N., Li, Y., Chen, L., Huang, X., Lui, S., et al. (2014). Intrinsic brain abnormalities in attention deficit hyperactivity disorder: a resting-state functional $\mathrm{mr}$ imaging study. Radiology, 272(2). doi: 10.1148/radiol.14131622

Lindfors, P., Berntsson, L., \& Lundberg, U. (2006). Factor structure of Ryff's psychological well-being scales in Swedish female and male whitecollar workers. Personality and Individual Differences, 40(6), 12131222.

Liu, F., Guo, W., Liu, L., Long, Z., Ma, C., Xue, Z., et al. (2013). Abnormal amplitude low-frequency oscillations in medication-naive, first-episode patients with major depressive disorder: A resting-state fMRI study. Journal of Affective Disorders, 146(3), 401-406.

Liu, Y., Wang, K., Yu, C., He, Y., Zhou, Y., Liang, M., et al. (2008). Regional homogeneity, functional connectivity and imaging markers of Alzheimer's disease: A review of resting-state fMRI studies. Neuropsychologia, 46(6), 1648-1656.

Luo, Y., Huang, X., Yang, Z., Li, B., Liu, J., \& Wei, D. (2014). Regional homogeneity of intrinsic brain activity in happy and unhappy individuals. PloS One, 9(1), e85181.

Lyubomirsky, S., Sheldon, K. M., \& Schkade, D. (2005). Pursuing happiness: The architecture of sustainable change. Review of General Psychology, 9(2), 111-131.

Mennes, M., Zuo, X.-N., Kelly, C., Di Martino, A., Zang, Y.-F., Biswal, B., et al. (2011). Linking inter-individual differences in neural activation and behavior to intrinsic brain dynamics. NeuroImage, 54(4), 2950-2959.

Mobbs, D., Hagan, C. C., Azim, E., Menon, V., \& Reiss, A. L. (2005). Personality predicts activity in reward and emotional regions associated with humor. Proceedings of the National Academy of Sciences, 102(45), 16502-16506.

Murphy, K., Birn, R. M., Handwerker, D. A., Jones, T. B., \& Bandettini, P. A. (2009). The impact of global signal regression on resting state correlations: Are anti-correlated networks introduced? NeuroImage, 44(3), 893-905.

Northoff, G., \& Panksepp, J. (2008). The trans-species concept of self and the subcortical-cortical midline system. Trends in Cognitive Sciences, 12(7), 259-264.

Omura, K., Constable, R. T., \& Canli, T. (2005). Amygdala gray matter concentration is associated with extraversion and neuroticism. Neuroreport, 16(17), 1905-1908.

Paulus, M. P., Rogalsky, C., Simmons, A., Feinstein, J. S., \& Stein, M. B. (2003). Increased activation in the right insula during risk-taking decision making is related to harm avoidance and neuroticism. NeuroImage, 19(4), 1439-1448.

Platek, S. M., \& Kemp, S. M. (2009). Is family special to the brain? An event-related fMRI study of familiar, familial, and self-face recognition. Neuropsychologia, 47(3), 849-858.

Preacher, K. J., \& Hayes, A. F. (2008). Asymptotic and resampling strategies for assessing and comparing indirect effects in multiple mediator models. Behavior Research Methods, 40(3), 879-891.

Raichle, M. E. (2010). Two views of brain function. Trends in Cognitive Sciences, 14(4), 180-190.

Ryan, R. M., \& Deci, E. L. (2001). On happiness and human potentials: A review of research on hedonic and eudaimonic well-being. Annual Review of Psychology, 52(1), 141-166.

Ryan, R. M., Huta, V., \& Deci, E. L. (2008). Living well: A selfdetermination theory perspective on eudaimonia. Journal of Happiness Studies, 9(1), 139-170.

Ryff, C. D. (1989). Happiness is everything, or is it? Explorations on the meaning of psychological well-being. Journal of Personality and Social Psychology, 57(6), 1069.

Ryff, C., Almeida, D. M., Ayanian, J. S., Carr, D. S., Cleary, P. D., Coe, C., et al. (2007). Midlife Development in the United States (MIDUS II), 2004-2006. Ann Arbor, MI: Inter-university Consortium for Political and Social Research (ICPSR).

Ryff, C. D., \& Keyes, C. L. M. (1995). The structure of psychological well-being revisited. Journal of Personality and Social Psychology, 69(4), 719

Saad, Z. S., Gotts, S. J., Murphy, K., Chen, G., Jo, H. J., Martin, A., et al. (2012). Trouble at rest: How correlation patterns and group differences become distorted after global signal regression. Brain Connectivity, 2(1), 25-32.

Schmutte, P. S., \& Ryff, C. D. (1997). Personality and well-being: Reexamining methods and meanings. Journal of Personality and Social Psychology, 73(3), 549.

Servaas, M. N., van der Velde, J., Costafreda, S. G., Horton, P., Ormel, J., Riese, H., et al. (2013). Neuroticism and the brain: A quantitative meta-analysis of neuroimaging studies investigating emotion processing. Neuroscience and Biobehavioral Reviews, 37(8), 15181529.

Sherman, S. M., \& Guillery, R. (2006). Exploring the thalamus and its role in cortical function. Cambridge, MA: MIT Press.

Singer, T., Critchley, H. D., \& Preuschoff, K. (2009). A common role of insula in feelings, empathy and uncertainty. Trends in Cognitive Sciences, 13(8), 334-340.

Spreng, R. N., \& Grady, C. L. (2010). Patterns of brain activity supporting autobiographical memory, prospection, and theory of mind, and their relationship to the default mode network. Journal of Cognitive Neuroscience, 22(6), 1112-1123. 
Steel, P., Schmidt, J., \& Shultz, J. (2008). Refining the relationship between personality and subjective well-being. Psychological Bulletin, 134(1), 138.

Strauss, G. P., Sandt, A. R., Catalano, L. T., \& Allen, D. N. (2012). Negative symptoms and depression predict lower psychological well-being in individuals with schizophrenia. Comprehensive Psychiatry, 53(8), 1137-1144.

Suslow, T., Kugel, H., Reber, H., Bauer, J., Dannlowski, U., Kersting, A., et al. (2010). Automatic brain response to facial emotion as a function of implicitly and explicitly measured extraversion. Neuroscience, 167(1), 111-123.

Sylvester, C., Corbetta, M., Raichle, M., Rodebaugh, T., Schlaggar, B., Sheline, Y., et al. (2012). Functional network dysfunction in anxiety and anxiety disorders. Trends in Neurosciences, 35(9), 527-535.

Takeuchi, H., Taki, Y., Nouchi, R., Sekiguchi, A., Hashizume, H., Sassa, Y., et al. (2013). Resting state functional connectivity associated with trait emotional intelligence. NeuroImage, 83, 318-328.

Tsakiris, M., Hesse, M. D., Boy, C., Haggard, P., \& Fink, G. R. (2007). Neural signatures of body ownership: A sensory network for bodily self-consciousness. Cerebral Cortex, 17(10), 2235-2244.

Van Dijk, K. R., Sabuncu, M. R., \& Buckner, R. L. (2012). The influence of head motion on intrinsic functional connectivity MRI. Neurolmage, 59(1), 431-438.

van Reekum, C. M., Urry, H. L., Johnstone, T., Thurow, M. E., Frye, C. J., Jackson, C. A., et al. (2007). Individual differences in amygdala and ventromedial prefrontal cortex activity are associated with evaluation speed and psychological well-being. Journal of Cognitive Neuroscience, 19(2), 237-248.

Wan, Y., Zheng, X., \& Yu, X. (2010). SWB \& PWB: The integration of two well-being research orientation. Studies of Psychology and Behavior, 8(3), 190-194.

Wang, L., Dai, W., Su, Y., Wang, G., Tan, Y., Jin, Z., et al. (2012). Amplitude of low-frequency oscillations in first-episode, treatment-naive patients with major depressive disorder: A restingstate functional MRI study. PloS One, 7(10), e48658.

Wang, X., Han, Z., He, Y., Caramazza, A., Song, L., \& Bi, Y. (2013). Where color rests: Spontaneous brain activity of bilateral fusiform and lingual regions predicts object color knowledge performance. NeuroImage, 76, 252-263.

Wei, L., Duan, X., Zheng, C., Wang, S., Gao, Q., Zhang, Z., et al. (2014). Specific frequency bands of amplitude low-frequency oscillation encodes personality. Human Brain Mapping, 35(1), 331-339.

Wei, T., Liang, X., He, Y., Zang, Y., Han, Z., Caramazza, A., et al. (2012). Predicting conceptual processing capacity from spontaneous neuronal activity of the left middle temporal gyrus. The Journal of Neuroscience, 32(2), 481-489.

Xu, J., Rees, G., Yin, X., Song, C., Han, Y., Ge, H., et al. (2014). Spontaneous neuronal activity predicts intersubject variations in executive control of attention. Neuroscience, 263, 181-192.

Yu, R., Chien, Y. L., Wang, H. L. S., Liu, C. M., Liu, C. C., Hwang, T. J., et al. (2014). Frequency-specific alternations in the amplitude of low-frequency fluctuations in schizophrenia. Human Brain Mapping, 35(2), 627-637.

Zeidan, F., Martucci, K. T., Kraft, R. A., Gordon, N. S., McHaffie, J. G., \& Coghill, R. C. (2011). Brain mechanisms supporting the modulation of pain by mindfulness meditation. Journal of Neuroscience, 31(14), 5540-5548.

Zhao, Z.-L., Fan, F.-M., Lu, J., Li, H.-J., Jia, L.-F., Han, Y., et al. (2014). Changes of gray matter volume and amplitude of low-frequency oscillations in amnestic MCI: An integrative multi-modal MRI study. Acta Radiologica, 0284185114533329.

Zhu, Q., Zhang, J., Luo, Y. L., Dilks, D. D., \& Liu, J. (2011). Resting-state neural activity across face-selective cortical regions is behaviorally relevant. The Journal of Neuroscience, 31(28), 10323-10330.

Zilbovicius, M., Meresse, I., Chabane, N., Brunelle, F., Samson, Y., \& Boddaert, N. (2006). Autism, the superior temporal sulcus and social perception. Trends in Neurosciences, 29(7), 359-366.

Zou, Q.-H., Zhu, C.-Z., Yang, Y., Zuo, X.-N., Long, X.-Y., Cao, Q.-J., et al. (2008). An improved approach to detection of amplitude of low-frequency fluctuation (ALFF) for resting-state fMRI: Fractional ALFF. Journal of Neuroscience Methods, 172(1), 137-141.

Zuo, X.-N., Di Martino, A., Kelly, C., Shehzad, Z. E., Gee, D. G., Klein, D. F., et al. (2010). The oscillating brain: Complex and reliable. NeuroImage, 49(2), 1432-1445. 